\title{
On Characterizations of McLLoG, ELLoGW, PTHL and k-GE Distributions
}

\author{
G.G. Hamedani \\ Department of Mathematics, Statistics and Computer Science \\ Marquette University, Milwaukee, WI 53201-1881 \\ gholamhoss.hamedani@marquette.edu \\ Nadeem Shafique But \\ Department of Family anf Community Medicine, \\ Rabigh Faculty of Medicine, King Abdulaziz Univerisy \\ nshafique@kau.edu.sa
}

\begin{abstract}
Huang S. and Oluyede (2016), Oluyede et al. (2016), Krishnarani (2016) and Rather and Rather (2017) consider the "McDonald Log-Logistic", the "Exponentiated Log-Logistic Weibull", the "Power Transformation Half-Logistic" and "k-Generalized Exponential" distributions, respectively, and study certain properties and applications of these distributions. The present short note is intended to complete, in some way, the above mentioned works via establishing certain characterizations of these distributions in different directions.
\end{abstract}

\section{Introduction}

Characterizations of distributions is an important research area which has recently attracted the attention of many researchers. This short note deals with various characterizations of the McDonald Log-Logistic (McLLoG), the Exponentiated LogLogistic Weibull (ELLoWG), the Power Transformation Half-Logistic (PTHL) and the kGeneralized Exponential (k-GE) distributions to complete, in some way, the above mentioned works. These characterizations are based on: $(i)$ a simple relationship between two truncated moments; ( $i i)$ the hazard function; (iii) reverse hazard function and (iv) conditional expectation of a function of the random variable. It should be mentioned that for characterization ( $i$ ) the $c d f$ (cumulative distribution function) is not required to have a closed form.

Huang S. and Oluyede (2016) introduced the McLLoG distribution with $c d f$ and $p d f$ (probability density function) given, respectively, by

$$
F(x ; a, b, c, \lambda, \gamma)=\frac{1}{B\left(a c^{-1}, b\right)} \int_{0}^{\left(1+\lambda x^{-\gamma}\right)} w^{a c^{-1}-1}(1-w)^{b-1}, x \geq 0,
$$

and

$$
\begin{aligned}
& f(x ; a, b, c, \lambda, \gamma) \\
& =\frac{c \lambda \gamma}{B\left(a c^{-1}, b\right)} x^{-\gamma-1}\left(1+\lambda x^{-\gamma}\right)^{a-1}\left[1-\left(1+\lambda x^{-\gamma}\right)^{-c}\right]^{b-1}, x>0,
\end{aligned}
$$

where $a, b, c, \lambda, \gamma$ are all positive parameters.

Oluyede et al. (2016) proposed the ELLoWG distribution with $c d f$ and $p d f$ given, respectively, by

$$
F(x ; \alpha, \beta, \gamma, c, s)=\left[1-\left(1+\left(\frac{x}{s}\right)^{c}\right)^{-1} e^{-\alpha x^{\beta}}\right]^{\gamma}, x \geq 0,
$$


and

$$
\begin{aligned}
& f(x ; \alpha, \beta, \gamma, c, s)=\gamma\left[1-\left(1+\left(\frac{x}{s}\right)^{c}\right)^{-1} e^{-\alpha x^{\beta}}\right]^{\gamma-1} \times \\
& e^{-\alpha x^{\beta}}\left(1+\left(\frac{x}{s}\right)^{c}\right)^{-1}\left\{\alpha \beta x^{\beta-1}+\frac{c x^{c-1}}{s^{c}+x^{c}}\right\},
\end{aligned}
$$

$x>0$, where $\alpha, \beta, \gamma, c, s$ are positive parameters.

Krishnarani (2016) introduced the PTHL distribution with $c d f$ and $p d f$ given, respectively, by

$$
F(x ; \alpha, \beta)=1-2\left(1+e^{\beta x^{\alpha}}\right)^{-1}, x \geq 0
$$

and

$$
f(x ; \alpha, \beta)=2 \alpha \beta x^{\alpha-1} e^{\beta x^{\alpha}}\left(1+e^{\beta x^{\alpha}}\right)^{-2}, x>0,
$$

where $\alpha, \beta$ are positive parameters.

Rather and Rather [8] proposed four almost similar GE distributions. We mention here the most general one and call it k-GE distribution with $c d f$ and $p d f$ given, respectively, by

$$
F(x ; \alpha, \beta, k)=\left(1-e^{-\beta x^{k}}\right)^{\alpha}, x \geq 0,
$$

and

$$
f(x ; \alpha, \beta)=k \alpha \beta x^{k-1} e^{-\beta x^{k}}\left(1-e^{-\beta x^{k}}\right)^{\alpha-1}, x>0,
$$

where $\alpha, \beta, k$ are positive parameters.

Remark 1.1. The $c d f(1.7)$ is a special case of the distribution proposed by Al-babtain et al. (2017). We believe that Rather and Rather were not aware of the work by Albabtain et al. (2017). The present author has characterized the $c d f$ of Al-babtain et al. (2017) earlier and therefore there is no need to characterize its special case here.

\section{Characterizations of McLLoG, ELLoGW and PTHL distributions}

We present our characterizations $(i)-(i v)$ in four subsections.

\subsection{Characterizations based on two truncated moments}

In this subsection we present characterizations of McLLoG, ElloGW and PTHL distributions in terms of a simple relationship between two truncated moments. The first characterization result employs a theorem due to Glänzel (1987), see Theorem 2.1.1 below. Note that the result holds also when the interval $H$ is not closed. Moreover, as mentioned above, it could be also applied when the $c d f F$ does not have a closed form. As shown in Glänzel (1990), this characterization is stable in the sense of weak convergence.

Theorem 2.1.1. Let $(\Omega, \mathcal{F}, \mathbf{P})$ be a given probability space and let $H=[d, e]$ be an interval for some $d<e \quad(d=-\infty, e=\infty$ mightaswellbeallowed). Let $X: \Omega \rightarrow H$ be a continuous random variable with the distribution function $F$ and let $g$ and $h$ be two real functions defined on $H$ such that

$$
\mathbf{E}[g(X) \mid X \geq x]=\mathbf{E}[h(X) \mid X \geq x] \xi(x), \quad x \in H,
$$


is defined with some real function $\eta$. Assume that $g, h \in C^{1}(H), \xi \in C^{2}(H)$ and $F$ is twice continuously differentiable and strictly monotone function on the set $H$. Finally, assume that the equation $\xi h=g$ has no real solution in the interior of $H$. Then $F$ is uniquely determined by the functions $g, h$ and $\xi$, particularly

$$
F(x)=\int_{a}^{x} C\left|\frac{\xi^{\prime}(u)}{\xi(u) h(u)-g(u)}\right| \exp (-s(u)) d u,
$$

where the function $s$ is a solution of the differential equation $s^{\prime}=\frac{\xi^{\prime} h}{\xi h-g}$ and $C$ is the normalization constant, such that $\int_{H} d F=1$.

Proposition 2.1.1. Let $X: \Omega \rightarrow(0, \infty)$ be a continuous random variable and let, $h(x)=$ $\left[1-\left(1+\lambda x^{-\gamma}\right)^{-c}\right]^{1-b}$ and $g(x)=h(x)\left(1+\lambda x^{-\gamma}\right)^{a}$ for $x>0$. The random variable $X$ has $p d f(1.2)$ if and only if the function $\xi$ defined in Theorem 2.1.1 has the form

$$
\xi(x)=\frac{1}{2}\left\{\left(1+\lambda x^{-\gamma}\right)^{a}+1\right\}, \quad x>0 .
$$

Proof. Let $X$ be a random variable with $p d f$ (1.2), then

and

$$
(1-F(x)) E[h(X) \mid X \geq x]=\frac{c}{a B\left(a c^{-1}, b\right)}\left\{\left(1+\lambda x^{-\gamma}\right)^{a}-1\right\}, \quad x>0,
$$

and finally

$$
(1-F(x)) E[g(X) \mid X \geq x]=\frac{c}{2 a B\left(a c^{-1}, b\right)}\left\{\left(1+\lambda x^{-\gamma}\right)^{2 a}-1\right\}, \quad x>0,
$$

$$
\xi(x) h(x)-g(x)=-\frac{1}{2} h(x)\left\{1-\left(1+\lambda x^{-\gamma}\right)^{a}\right\}<0 \quad \text { for } x>0 .
$$

Conversely, if $\xi$ is given as above, then

and hence

$$
s^{\prime}(x)=\frac{\xi^{\prime}(x) h(x)}{\xi(x) h(x)-g(x)}=\frac{a \lambda \gamma x^{-\gamma-1}\left(1+\lambda x^{-\gamma}\right)^{a-1}}{1-\left(1+\lambda x^{-\gamma}\right)^{a}} \quad x>0,
$$

$$
s(x)=\log \left\{1-\left(1+\lambda x^{-\gamma}\right)^{a}\right\}, \quad x>0 .
$$

Now, in view of Theorem 2.1.1, $X$ has density (1.2).

Corollary 2.1.1. Let $X: \Omega \rightarrow(0, \infty)$ be a continuous random variable and let $h(x)$ be as in Proposition 2.1.1. The pdf of $X$ is (1.2) if and only if there exist functions $g$ and $\xi$ defined in Theorem 2.1.1 satisfying the differential equation

$$
\frac{\xi^{\prime}(x) h(x)}{\xi(x) h(x)-g(x)}=\frac{a \lambda \gamma x^{-\gamma-1}\left(1+\lambda x^{-\gamma}\right)^{a-1}}{1-\left(1+\lambda x^{-\gamma}\right)^{a}}, \quad x>0 .
$$

The general solution of the differential equation in Corollary 2.1.1 is

$$
\xi(x)=\left\{1-\left(1+\lambda x^{-\gamma}\right)^{a}\right\}^{-1}\left[-\int a \lambda \gamma x^{-\gamma-1}\left(1+\lambda x^{-\gamma}\right)^{a-1}(h(x))^{-1} g(x)+D\right],
$$

where $D$ is a constant. Note that a set of functions satisfying the above differential equation is given in Proposition 2.1.1 with $D=\frac{1}{2}$. However, it should be also noted that there are other triplets $(h, g, \xi)$ satisfying the conditions of Theorem 2.1.1. 
Proposition 2.1.2. Let $X: \Omega \rightarrow(0, \infty)$ be a continuous random variable and let, $h(x)=$ $\left[1-\left(1+\left(\frac{x}{s}\right)^{c}\right)^{-1} e^{-\alpha x^{\beta}}\right]^{1-\gamma}$ and $g(x)=h(x)\left(1+\left(\frac{x}{s}\right)^{c}\right)^{-1} e^{-\alpha x^{\beta}} \quad$ for $x>0$. The random variable $X$ has $p d f(1.4)$ if and only if the function $\xi$ defined in Theorem 2.1.1 has the form

$$
\xi(x)=\frac{1}{2}\left(1+\left(\frac{x}{S}\right)^{c}\right)^{-1} e^{-\alpha x^{\beta}}, \quad x>0 .
$$

Proof. Let $X$ be a random variable with $p d f$ (1.4), then

and

$$
(1-F(x)) E[h(X) \mid X \geq x]=\gamma\left(1+\left(\frac{x}{S}\right)^{c}\right)^{-1} e^{-\alpha x^{\beta}}, \quad x>0
$$

and finally

$$
(1-F(x)) E[g(X) \mid X \geq x]=\frac{\gamma}{2}\left(1+\left(\frac{x}{S}\right)^{c}\right)^{-2} e^{-2 \alpha x^{\beta}}, \quad x>0,
$$

$$
\xi(x) h(x)-g(x)=-\frac{1}{2} h(x)\left(1+\left(\frac{x}{s}\right)^{c}\right)^{-1} e^{-\alpha x^{\beta}}<0 \quad \text { for } x>0 .
$$

Conversely, if $\xi$ is given as above, then

$$
s^{\prime}(x)=\frac{\xi^{\prime}(x) h(x)}{\xi(x) h(x)-g(x)}=\alpha \beta x^{\beta-1}+\frac{c x^{c-1}}{s^{c}+x^{c}} \quad x>0 .
$$

Now, in view of Theorem 2.1.1, $X$ has density (1.4).

Corollary 2.1.2. Let $X: \Omega \rightarrow(0, \infty)$ be a continuous random variable and let $h(x)$ be as in Proposition 2.1.2. The pdf of $X$ is (1.4) if and only if there exist functions $g$ and $\xi$ defined in Theorem 2.1.1 satisfying the differential equation

$$
\frac{\xi^{\prime}(x) h(x)}{\xi(x) h(x)-g(x)}=\alpha \beta x^{\beta-1}+\frac{c x^{c-1}}{s^{c}+x^{c}}, \quad x>0 .
$$

The general solution of the differential equation in Corollary 2.1.2 is

$$
\xi(x)=\left(1+\left(\frac{x}{s}\right)^{c}\right) e^{\alpha x^{\beta}}\left[\begin{array}{l}
-\int\left\{\alpha x^{\beta-1}+\frac{c x^{c-1}}{s^{c}+x^{c}}\right\} \times \\
\left(1+\left(\frac{x}{s}\right)^{c}\right)^{-1} e^{-\alpha x^{\beta}}(h(x))^{-1} g(x)+D
\end{array}\right],
$$

where $D$ is a constant. Note that a set of functions satisfying the above differential equation is given in Proposition 2.1.2 with $D=\frac{1}{2}$. However, it should be also noted that there are other triplets $(h, g, \xi)$ satisfying the conditions of Theorem 2.1.1.

Proposition 2.1.3. Let $X: \Omega \rightarrow(0, \infty)$ be a continuous random variable and let $h(x) \equiv$ 1 and $g(x)=\left(1+e^{\beta x^{\alpha}}\right)^{-1}$ for $x>0$. The random variable $X$ has $p d f(1.6)$ if and only if the function $\xi$ defined in Theorem 2.1.1 has the form

$$
\xi(x)=\frac{1}{2}\left(1+e^{\beta x^{\alpha}}\right)^{-1}, \quad x>0 .
$$


Proof. Let $X$ be a random variable with $p d f$ (1.6), then

and

$$
(1-F(x)) E[h(X) \mid X \geq x]=2\left(1+e^{\beta x^{\alpha}}\right)^{-1}, x>0 \text {, }
$$

and finally

$$
(1-F(x)) E[g(X) \mid X \geq x]=\left(1+e^{\beta x^{\alpha}}\right)^{-2}, \quad x>0,
$$

$$
\xi(x) h(x)-g(x)=-\frac{1}{2}\left(1+e^{\beta x^{\alpha}}\right)^{-1}<0 \text { for } x>0 .
$$

Conversely, if $\xi$ is given as above, then

$$
s^{\prime}(x)=\frac{\xi^{\prime}(x) h(x)}{\xi(x) h(x)-g(x)}=\frac{\alpha \beta x^{\alpha-1} e^{\beta x^{\alpha}}}{\left(1+e^{\beta x^{\alpha}}\right)}, \quad x>0 .
$$

Now, in view of Theorem 2.1.1, $X$ has density (1.6).

Corollary 2.1.3. Let $X: \Omega \rightarrow(0, \infty)$ be a continuous random variable and let $h(x)$ be as in Proposition 2.1.3. The pdf of $X$ is (1.6) if and only if there exist functions $g$ and $\xi$ defined in Theorem 2.1.1 satisfying the differential equation

$$
\frac{\xi^{\prime}(x) h(x)}{\xi(x) h(x)-g(x)}=\frac{\alpha \beta x^{\alpha-1} e^{\beta x^{\alpha}}}{\left(1+e^{\beta x^{\alpha}}\right)}, \quad x>0 .
$$

The general solution of the differential equation in Corollary 2.1.3 is

$$
\xi(x)=\left(1+e^{\beta x^{\alpha}}\right)\left[-\int \alpha \beta x^{\alpha-1} e^{\beta x^{\alpha}}\left(1+e^{\beta x^{\alpha}}\right)^{-2}(h(x))^{-1} g(x)+D\right],
$$

where $D$ is a constant. Note that a set of functions satisfying the above differential equation is given in Proposition 2.1.3 with $D=0$. However, it should be also noted that there are other triplets $(h, g, \xi)$ satisfying the conditions of Theorem 2.1.1.

\subsection{Characterization based on hazard function}

It is known that the hazard function, $h_{F}$, of a twice differentiable distribution function, $F$, satisfies the first order differential equation

$$
\frac{f^{\prime}(x)}{f(x)}=\frac{h_{F}^{\prime}(x)}{h_{F}(x)}-h_{F}(x) \text {. }
$$

For many univariate continuous distributions, this is the only characterization available in terms of the hazard function. The following characterizations establish non-trivial characterizations of McLLoG distribution, for $a=c$, ELLoGW distribution, for $\alpha=$ $0, \gamma=1$ and PTHL in terms of the hazard function, which are not of the above trivial form.

Proposition 2.2.1. Let $X: \Omega \rightarrow(0, \infty)$ be a continuous random variable. The $p d f$ of $X$ is (1.2), for $a=c$, if and only if its hazard function $h_{F}(x)$ satisfies the differential equation

$$
h_{F}^{\prime}(x)+\frac{\gamma+1}{x} h_{F}(x)=a b \lambda \gamma x^{-\gamma-1} \frac{d}{d x}\left\{\frac{\left(1+\lambda x^{-\gamma}\right)^{-a-1}}{1-\left(1+\lambda x^{-\gamma}\right)^{-a}}\right\}, x>0 .
$$


Proof. If $X$ has $p d f$ (1.2), then clearly the above differential equation holds. Now, if the differential equation holds, then

$$
\frac{d}{d x}\left\{\left(x^{\gamma+1} h_{F}(x)\right\}=a b \lambda \gamma \frac{d}{d x}\left\{\frac{\left(1+\lambda x^{-\gamma}\right)^{-a-1}}{1-\left(1+\lambda x^{-\gamma}\right)^{-a}}\right\}\right.
$$

or

$$
h_{F}(x)=\frac{a b \lambda \gamma x^{-\gamma-1}\left(1+\lambda x^{-\gamma}\right)^{-a-1}}{1-\left(1+\lambda x^{-\gamma}\right)^{-a}}, \quad x>0,
$$

which is the hazard function of the McLLoG distribution, for $a=c$.

Proposition 2.2.2. Let $X: \Omega \rightarrow(0, \infty)$ be a continuous random variable. The $p d f$ of $X$ is (1.4), for $\alpha=0, \gamma=1$, if and only if its hazard function $h_{F}(x)$ satisfies the differential equation

$$
h_{F}^{\prime}(x)-\frac{c-1}{x} h_{F}(x)=-c^{2} s^{-2}\left(\frac{x}{s}\right)^{2(c-1)}\left(1+\left(\frac{x}{s}\right)^{c}\right)^{-2}, x>0 .
$$

Proof. Is similar to the proof of Proposition 2.2.1 and hence omitted.

Proposition 2.2.3. Let $X: \Omega \rightarrow(0, \infty)$ be a continuous random variable. The $p d f$ of $X$ is (1.6) if and only if its hazard function $h_{F}(x)$ satisfies the differential equation

$$
h_{F}^{\prime}(x)-\alpha \beta x^{\alpha-1} h_{F}(x)=\alpha \beta x^{\alpha-1} e^{\beta x^{\alpha}}\left\{\frac{\alpha-1}{1+e^{\beta x^{\alpha}}}-\frac{\alpha \beta x^{\alpha-1}}{\left(1+e^{\beta x^{\alpha}}\right)^{2}}\right\}, x>0 .
$$

Proof. Is similar to the proof of Proposition 2.2.1 and hence omitted.

\subsection{Characterization in terms of the reverse hazard function}

The reverse hazard function, $r_{F}$, of a twice differentiable distribution function, $F$, is defined as

$$
r_{F}(x)=\frac{f(x)}{F(x)}, \quad x \in \text { supportof } F
$$

Proposition 2.3.1. Let $X: \Omega \rightarrow(0, \infty)$ be a continuous random variable. The pdf of $X$ is (1.2), for $b=1$, if and only if its reverse hazard function $r_{F}(x)$ satisfies the differential equation

$$
r_{F}^{\prime}(x)+\frac{\gamma+1}{x} r_{F}(x)=\frac{a \lambda^{2} \gamma^{2} x^{-2(\gamma+1)}}{\left(1+\lambda x^{-\gamma}\right)^{2}}, \quad x>0
$$

Proof. Is similar to the proof of Proposition 2.2.1 and hence omitted.

Proposition 2.3.1. Let $X: \Omega \rightarrow(0, \infty)$ be a continuous random variable. The pdf of $X$ is (1.4) if and only if its reverse hazard function $r_{F}(x)$ satisfies the differential equation

$$
r_{F}^{\prime}(x)+\alpha \beta x^{\beta-1} r_{F}(x)=\gamma e^{-\alpha x^{\beta}} \frac{d}{d x}\left\{\frac{\left(1+\left(\frac{x}{s}\right)^{c}\right)^{-1}\left\{\alpha \beta x^{\beta-1}+\frac{c x^{c-1}}{s^{c}+x^{c}}\right\}}{1-\left(1+\left(\frac{x}{s}\right)^{c}\right)^{-1} e^{-\alpha x^{\beta}}}\right\}, \quad x>0 .
$$

Proof. Is similar to the proof of Proposition 2.2.1 and hence omitted. 


\subsection{Characterizations Based on Conditional Expectation}

The following propositions have already appeared in Hamedani (2013), so we will just state them here which can be used to characterize the McLLoG and ElloGW distributions.

Proposition 2.4.1. Let $X: \Omega \rightarrow(e, f)$ be a continuous random variable with $c d f F$. Let $\psi(x)$ be a differentiable function on $(e, f)$ with $\lim _{x \rightarrow e^{+}} \psi(x)=1$. Then for $\delta \neq$ 1 ,

$$
E[\psi(X) \mid X \geq x]=\delta \psi(x), \quad x \in(e, f),
$$

if and only if

$$
\psi(x)=(1-F(x))^{\frac{1}{\delta}-1}, \quad x \in(e, f) .
$$

Proposition 2.4.2. Let $X: \Omega \rightarrow(e, f)$ be a continuous random variable with $c d f F$. Let $\psi_{1}(x)$ be a differentiable function on $(e, f)$ with $\lim _{x \rightarrow f^{-}} \psi_{1}(x)=1$. Then for $\delta_{1} \neq 1$,

$$
E\left[\psi_{1}(X) \mid X \leq x\right]=\delta \psi_{1}(x), \quad x \in(e, f),
$$

implies

$$
\psi_{1}(x)=(F(x))^{\frac{1}{\delta}-1}, \quad x \in(e, f)
$$

Remarks 2.4.1. (A) For $(e, f)=(0, \infty), a=c, \psi(x)=1-\left(1+\lambda x^{-\gamma}\right)^{-c}$ and $\delta=$ $\frac{b}{b+1}$, Proposition 2.4.1 provides a characterization of MclloG distribution. (B) For $(e, f)=(0, \infty), \gamma=1, \psi(x)=\left(1+\left(\frac{x}{s}\right)^{c}\right)^{-1 / \alpha} e^{-x^{\beta}}$ and $\delta=\frac{\alpha}{\alpha+1}$, Proposition 2.4.1 provides a characterization of ELLoGW distribution. $(C)$ For $(e, f)=(0, \infty), b=1$, $\psi_{1}(x)=\left(1+\lambda x^{-\gamma}\right)^{-1}$ and $\delta_{1}=\frac{a}{a+1}$, Proposition 2.4.2 provides a characterization of McLLoG distribution. (D) For $(e, f)=(0, \infty), \psi_{1}(x)=1-\left(1+\left(\frac{x}{s}\right)^{c}\right)^{-1} e^{-\alpha x^{\beta}}$ and $\delta_{1}=\frac{\gamma}{\gamma+1}$, Proposition 2.4.2 provides a characterization of ELLoGW distribution. $(E)$ For $(e, f)=(0, \infty), \psi(x)=\sqrt{2}\left(1+e^{\beta x^{\alpha}}\right)^{-1 / 2}$ and $\delta=\frac{1}{2}$, Proposition 2.4.1 provides a PTHL distribution. Of course there are other suitable functions than the ones we mentioned above, which are chosen for simplicity.

\section{References}

1. Al-Babtain, A., Fattah, A. A., Ahmed, A.-H. N., \& Merovci, F. (2017). The Kumaraswamy-transmuted exponentiated modified Weibull distribution. Communications in Statistics - Simulation and Computation, 46(5), 3812-3832. http://doi.org/10.1080/03610918.2015.1011338

2. Glanzel, W. (1990). Some consequences of a characterization theorem based on truncated moments. Statistics, 21(4), 613-618. http://doi.org/10.1080/02331889008802273 
3. Glänzel, W. (1987). A Characterization Theorem Based on Truncated Moments and its Application to Some Distribution Families. In Mathematical Statistics and Probability Theory (pp. 75-84). Dordrecht: Springer Netherlands. http://doi.org/10.1007/978-94-009-3965-3_8

4. Hamedani, G. G. (2013). On certain generalized gamma convolution distributions II. Technical Report, No. 484, MSCS,Marquette University.

5. Huang, S. and Oluyede, B. O. (2016). The McDonald log-logistic distribution with applications to lifetime and pricing data. J.of Probability and Statistics, 14(2), 123-139.

6. Krishnarani, S. D. (2016). On a Power Transformation of Half-Logistic Distribution. Journal of Probability and Statistics, 2016, 10 Pages. http://doi.org/10.1155/2016/2084236

7. Oluyede, B.O., Basele, G., Makubate, B. and Huang, S. (2016). A new class of generalized log-logistic Weibull distribution: theory, properties and applications. J.of Probability and Statistics, 14(2), 171-201.

8. Rather, N. A., \& Rather, T. A. (2017). New Generalizations of Exponential Distribution with Applications. Journal of Probability and Statistics, 2017, 9 Pages. http://doi.org/10.1155/2017/2106748. 